\title{
From the Editors' Desk: Sticking with the Script
}

\author{
Richard L. Kravitz, MD, MSPH \\ Division of General Medicine, University of California at Davis, Sacramento, CA, USA.
}

J Gen Intern Med 26(10): 1083

DOI: $10.1007 / \mathrm{s} 11606-011-1820-8$

(c) Society of General Internal Medicine 2011

$I^{n}$ n a 1990 Academic Medicine article subsequently cited almost 500 times, H.G. Schmidt and colleagues introduced the concept of illness scripts. Their work was motivated by the observation that experts solve clinical cases differently than novices. While novices reason pathophysiologically, experts match what they see and apprehend with cognitive structures that encode knowledge about the way a disease presents and evolves. These encoded patterns are called illness scripts. Fever, anemia and hematuria in an intravenous drug user: bacterial endocarditis. Chronic erythematous scale over the elbows combined with nail pitting: psoriasis.

In the course of a career, physicians acquire hundreds if not thousands of illness scripts.

Having a robust illness script cerebral repository serves two ends. First, scripts are available for matching to the available clinical facts. Equally important, scripts guide the acquisition of those facts. If you can't access an illness script for lupus that includes a nod to pericarditis, you may not notice that faint three-component scratchy sound as you move the stethoscope across the anterior chest of a young woman with low grade fever and malar rash.

This month in Exercises in Clinical Reasoning, Erickson et al. invoke several illness scripts as they walk us through a case of bilateral pleural effusion. The case has some surprising twists and turns, but the discussant remains flexible, invoking new illness scripts as it becomes obvious that the old ones don't fit the data. Clinicians will appreciate the opportunity to review the differential diagnosis of pleural effusion and to integrate the historical, physical, laboratory and echocardiographic findings. Clinician-educators may also like the attention given to "meta-cognition"- the italicized description of how the discussant thinks about the case, and in particular how various cognitive heuristics (including illness scripts) may lead to the right or wrong diagnosis.

Like doctors, patients also seek to understand the world, and they use similar heuristics (including scripts) to help them navigate the illness experience. Knowing more about how patients of different cultural backgrounds interpret clinical symptoms could help physicians devise more effective, culturally concordant treatment approaches. In a qualitative study of Latina survivors of domestic violence, Nicolaidis and colleagues report that focus group members saw depression as a consequence of "guardarse las cosas adentro" (keeping things inside). They suggest that physicians might help such patients by actively inquiring about life stressors and encouraging them to talk about their lives. The point is not to assume that Latinas, or anyone else, adheres to a particular life script. To paraphrase Hamlet, the awareness is all. Such awareness can lead to informed, empathic questioning and ultimately to better understanding of the patient as a person, not a cultural stereotype.

Sometimes doctors get stuck on script. A good example emerges in the article by Hall et al., which reports that in 2007-2008, 80\% of US primary care physicians talked with their male patients about prostate-specific antigen (PSA) screening, and of those, nearly two-thirds tried to talk their patients into getting the test. This report is sharply at odds with the academic consensus (emerging even in 2007-2008) that PSA screening ought to be a shared decision that accounts for patients' preferences and values. PSA testing is clearly right for some men and not for others, with the balance hinging on things like risk factors for prostate cancer, fear of dying from prostate cancer and aversion to the side effects of prostate cancer therapy. However, this message has not yet been incorporated into the dominant public health "script," which might be summarized as "screen early, screen often, collect HEDIS points." We can do better.

And sometimes we need to go completely off script. In Healing Arts, Terry Wahls bravely describes his own quest to identify effective interventions for a progressive neurological illness. By writing about his long (and partly successful) history of self-experimentation with diet, electrostimulation, vitamins and anti-oxidants, Wahls reminds us of the limits of evidence-based medicine and-at least in some carefully delimited circumstances-the value of pathophysiologically informed guesswork. Like actors, physicians can-and sometimes should-exercise creative license and think outside the box of current evidence. In losing the script, who knows? We may find hope for our patients and ourselves.

Corresponding Author: Richard L. Kravitz, MD, MSPH; Division of General Medicine, University of California at Davis, $4150 \mathrm{~V}$ Street, Suite 2400 PSSB, Sacramento, CA 95817, USA (e-mail: rlkravitz@ucdavis.edu). 